\title{
Effects of Cytoskeletal Orientations on Deformation of a Cell Residing in a Collagen Gel Construct*
}

\author{
Masanori NAKAMURA**, Yoshihiro UJIHARA***, Masatsugu SOGA****, \\ Kenichiro KOSHIYAMA****, Hiroshi MIYAZAKI**** and Shigeo WADA**** \\ ${ }^{* *}$ Graduate School of Science and Engineering, Saitama University, Saitama, Japan \\ E-mail: masanorin@mech.saitama-u.ac.jp

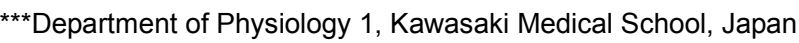 \\ ${ }^{* * * * G r a d u a t e}$ School of Engineering Science, Osaka University, Toyonaka, Osaka, Japan
}

\begin{abstract}
The effects of cytoskeleton orientation angle on strain transmission from a tissue to a cell and its internal cytoskeletons were investigated by using a model where a cell was integrated in a tissue that assumes a collagen gel construct. A cell with uni-directionally or randomly aligned cytoskeletons was embedded in a tissue, which was stretched to a strain of 0.1 . When the initial orientation angle of the cytoskeleton was zero, which corresponded to the stretch direction of the tissue, cell strain was minimal and mean cytoskeletal strain was maximal. As the initial cytoskeleton orientation angle increased, mean cytoskeletal strain values decreased, while cell strain increased. Cell strain decreased at an initial cytoskeleton orientation angle of $60^{\circ}$. At this angle, the mean cytoskeletal strain value was nearly zero, which was absolutely minimal. Subsequent increases in the initial cytoskeleton orientation angle resulted in further decreases in cell and cytoskeletal strain. The present multi-scale model may help in achieving structural integration of the biomechanical organization and provide valuable information for understanding the mechanisms underlying cellular remodeling.
\end{abstract}

Key words: Biomechanics, Cell, Cytoskeleton, Multi-Scale Modeling, Tissue

\section{Introduction}

Tissue remodeling takes place as a result of cellular responses ${ }^{(1)}$. In vitro studies of cell culture have shown that cells which are subjected to a change in their external mechanical environment exhibit various responses including an alteration of the shape ${ }^{(2)}$, orientation $^{(3),(4)}$, stiffness ${ }^{(5)}$, and viscoelastic properties ${ }^{(2)}$. A part of those responses was shown to depend on the applied direction ${ }^{(6),(7)}$ and magnitude of the mechanical stresses ${ }^{(8),(9)}$.

Recent studies have demonstrated that the cytoskeleton plays a key role in sensing mechanical stimuli and transforming these stimuli to intracellular signals ${ }^{(10)}$. The data suggest that the cellular responses to mechanical stimuli emerge as the collective responses of cytoskeletons. Among cytoskeletons, actin fibers are known to act as the main mechanosensing components with which cells sense mechanical force. Actin fibers orient along the major axis of a cell, and their orientation has significant effects on cellular responses $^{(7),(11)}$. For instance, Adachi et al. ${ }^{(7)}$ demonstrated that the sensitivity of osteoblastic

*Received 4 Oct., 2011 (№. 11-0600) [DOI: 10.1299/jbse.7.2]

Copyright $(\odot 2012$ by JSME 
cells to a mechanical stimulus depended on the angle of the applied deformation with respect to the actin fiber orientation.

On the basis of those findings, tissue engineering studies have become a rapidly growing area of research in bioengineering. The tissue engineering is aimed to construct functional tissues having mechanical and structural properties to repair or replace damaged tissues (i.e., bone, cartilage, skin etc.), for instance those having larger elastic modulus ${ }^{(12),(13)}$, by providing proper mechanical stimuli to cells or cytoskeletons in culture. The success of constructing functional tissues is to apply controlled mechanical loadings to developing constructs in an attempt to direct the development of a healthy and biomechanically-competent tissue, to enhance tissue functionality and to maximize the benefits from mechanical loading. In the development of a functional tissue engineering protocol, it is essential to understand how mechanical loading applied to the tissue is transmitted to a cell to generate the desired response. However, determination methodology of optimal loading protocols remains to rely on trials and errors, which is often quite inefficient and retards the development of tissue engineering technologies. Taking account of a wide variability in cellular functions, it is unlikely that a single loading regime is versatile for all cell types and scaffolds. Rather, a unique loading scheme that meets scaffold composition, cell density and cell conditions and so forth is required for each cell type. Moreover, changes in material properties of scaffolds by cell culture must also be considered to keep the optimal loading conditions for cells.

In attempts to study the mechanisms underlying cellular remodeling within a tissue and mechanotransduction from tissues to cells, measurements of tissue and nucleus strains have been conducted ${ }^{(14),(15)}$. It is however still challenging to quantitatively assess deformation of the cytoskeleton, cells, and tissues at the same time, based solely on experimental data. Therefore, we need to have a computational model in order to efficiently develop the optimal tissue engineering protocol that gives the type and magnitude of mechanical stimuli that are the most beneficial for developing constructs.

Our project is aimed at establishing a system that helps predict how loading applied to the tissue affects cell behavior and tissue development. Usually, particular approaches are used to describe the system at each scale. A continuum model is adopted for describing mechanics of tissues and cells. However, the continuum model may not work properly in the description of behaviors of intracellular elements because at this scale, distribution of elements is spatially discontinuous. Instead, discrete element models, or their alternative models are used. In fact, researchers involved in the study of each scale level look at a phenomenon with specific length and time, which also hinders integration of those models. Thus one of the critical issues in developing a multi-scale model is a bidirectional linking of models specified at each scale. One of the ways to resolve this problem is to model all elements on a unified principle of mechanics that work across all scales. In our project, we try to model the behaviors of cytoskeletons, a cell and a tissue on the basis of the minimum energy principle. In the previous study, we developed a computational model of a cell, i.e., "the mechano-cell model," which is capable of expressing the deformation behavior of a cell during a mechanical test ${ }^{(16)}$. The mechano-cell model is aimed at understanding how subcellular components passively alter their structure with cell deformation, and how such a change in subcellular components is reflected in the mechanical properties of cells. As a part of the project, we demonstrated that the global mechanical properties of a cell is affected by the initial orientation of cytoskeletons ${ }^{(17)}$. The next step is to create the model closer to the situation of tissue engineering where a cell is embedded in a tissue. Thus, in the present study, we integrated the cell model we developed in the previous studies ${ }^{(16),(17)}$, in a newly developed tissue model to create a multi-scale model that helps us understand how cytoskeletons behave upon tissue deformation. As the initial attempt of computational modeling of tissue engineering, here we consider a relatively easy situation where cell 
culture is just started. In this consideration, we assume the tissue as a collagen gel construct that is not so rich in collagen fibers and can be treated as an isotropic material. Using the model, we investigated the effects of initial cytoskeleton orientation on strain transmission from a biological tissue to a cell and its internal cytoskeletons.

\section{Methods}

\subsection{Modeling of a tissue}

A mechanical model of a tissue was established based on continuum mechanics. A cross section of the tissue geometry is presented in Fig. 1 for internal visualization. The tissue is cubic with each line having a length of $2 a$, or $100 \mu \mathrm{m}$. The tissue has a spherical hollow at its center in which a cell resides. For further modeling, a Cartesian coordinate system $(x, y$, $z$ ) was set at the origin $\mathrm{O}$, which was defined at the center of tissue (see Fig. 1). The tissue was divided into first-order tetrahedral meshes. As described in the introduction, here we assume the situation where a cell is just seeded in a tissue and collagen fibers in the gel are not aligned in a particular direction. Under this assumption, a tissue was regarded as a homogeneous isotropic material for simplicity. Its strain energy density function is given by

$$
W^{t^{*}}=\frac{1}{2} \boldsymbol{\sigma}: \mathbf{E}
$$

where $\sigma$ is a Cauchy stress tensor and $\mathrm{E}$ is a Green-Lagrange strain tensor. The Cauchy stress tensor is described by

$$
\boldsymbol{\sigma}=\frac{v E^{t}}{(1+v)(1-2 v)}(\operatorname{tr} \mathbf{E}) \mathbf{I}+\frac{E^{t}}{1+v} \mathbf{E}
$$

where $E^{t}$ is the Young's modulus of the tissue, $v$ is a Poisson ratio, and $\mathbf{I}$ is a unit tensor. The Green-Lagrange strain tensor is given by

$$
\mathbf{E}=\frac{1}{2}\left\{\frac{\partial \mathbf{u}}{\partial \mathbf{r}}+\left(\frac{\partial \mathbf{u}}{\partial \mathbf{r}}\right)^{T}+\frac{\partial \mathbf{u}}{\partial \mathbf{r}}\left(\frac{\partial \mathbf{u}}{\partial \mathbf{r}}\right)^{T}\right\}
$$

where $\mathbf{u}=\left(u_{x}, u_{y}, u_{z}\right)$ is a displacement vector, $\mathbf{r}=\left(r_{x}, r_{y}, r_{z}\right)$ is a positional vector, and superscript $T$ denotes the transpose of a vector. The total strain energy is thus determined by

$$
W^{t}=\sum_{i=1}^{N_{e}}\left(W^{t^{*}}\right)_{i} V_{i}
$$

where $\left(W^{t^{*}}\right)_{i}$ stands for $W^{t *}$ at element $i, V_{i}$ is the volume of $i$, and $N_{e}$ is the number of elements in the tissue model.

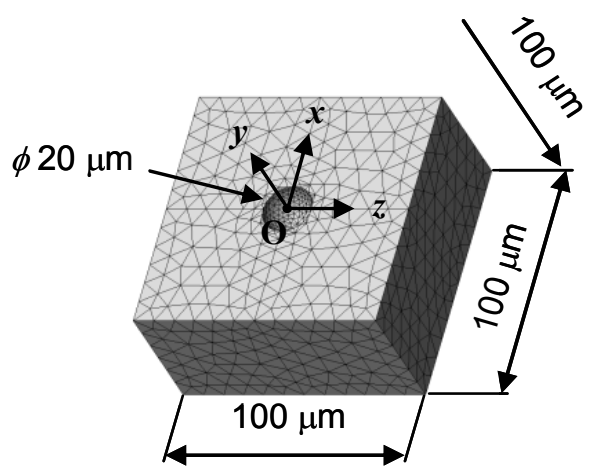

Fig. 1 Tissue model 


\subsection{Modeling of a cell}

The model developed in Ujihara et al. ${ }^{(16)}$ was used as a model of a cell residing in a tissue. Briefly, the model consists of three main components: a cell membrane (CM), a nuclear envelope (NE), and cytoskeletons (CSKs). Here, CM and NE were modeled together with underlying cytoskeletal networks. Note that elements on the CM were created using triangular elements at the hollowed portion of the tissue model, such that nodes and elements on the CM coincide with those on the tissue at their boundary (Fig. 2(a)). As a result, nodes at the boundary between the cell and tissue have characteristics of both the cell and tissue. In other words, there is no slip between the CM and the tissue even if they deform. This represents a biologically idealized situation where the CM is perfectly fixed to the extracellular matrix or tissue by focal adhesions through which the CSKs connect to the tissue. The mechanical nature of the $\mathrm{CM}$ and $\mathrm{NE}$ was expressed as a spherical membrane of spring networks. Figure 2(b) is a schematic drawing of the mechanical model of CM and NE where two elements with four nodes are presented. Nodes were linked by a spring (spring constant $k_{s}$ ) that provided resistance to in-plane deformation (stretch or compression). Neighboring elements were also connected with a spring (spring constant $k_{b}$ ) to resist element folding. The stretching energy, $W_{s}$ and bending energy, $W_{b}$ were modeled as

$$
\begin{aligned}
& W_{s}=\frac{1}{2} k_{s} \sum_{l=1}^{N_{s}}\left(L_{l}-L_{l 0}\right)^{2}, \\
& W_{b}=\frac{1}{2} k_{b} \sum_{l=1}^{N_{b}} L_{l} \tan ^{2}\left(\frac{\theta_{l}}{2}\right)
\end{aligned}
$$

where $k_{s}$ and $k_{b}$ represent spring constants, $N_{s}, N_{b}$ are the number of stretching springs and bending springs, $L_{l 0}$ and $L_{l}$ are the length of the spring in the natural state and after deformation, and $\theta_{l}$ is the contact angle between neighboring elements.

To express the resistance to change in surface area of $\mathrm{CM}$ and NE, we introduced potential energy functions for an area change. We defined a potential function for the change in whole area and local area. Mathematically, it was given by

$$
W_{A}=\frac{1}{2} k_{A}\left(\frac{A-A_{0}}{A_{0}}\right)^{2} A_{0}+\frac{1}{2} k_{a} \sum_{e=1}^{N_{e}^{c}}\left(\frac{A_{e}-A_{e 0}}{A_{e 0}}\right)^{2} A_{e 0},
$$

where $A$ and $A_{e}$ are the areas of the whole membrane and each element, respectively, subscript 0 denotes the natural state, $k_{A}$ and $k_{a}$ are coefficients for the whole and local area constraints, respectively, and $N_{e}^{c}$ is the number of elements.

Here we modeled a cytoskeletal element regardless of its kind (actin fibers, intermediate filaments and microtubules and so forth). Thus, the cytoskeletal element modeled here has characteristics of all sorts of cytoskeletal elements. A cytoskeletal element was represented by a spring that connects two nodes on the CM. On the basis of Hooke's law, a potential energy $W^{C S K}$ generated by a change in the length of CSKs was modeled as

$$
W^{C S K}=\frac{1}{2} k_{C S K} \sum_{i=1}^{N^{C S K}}\left(l_{i}-l_{0 i}\right)^{2}
$$

where $k_{C S K}$ is the spring constant of the CSK, $l_{0 i}$ and $l_{i}$ are the length of $\mathrm{CSK}_{i}$ at the natural state and after deformation, respectively, and $N^{C S K}$ is the total number of CSKs.

The cytoplasm resides between the CM and NE. In accordance with the deformation of the $\mathrm{CM}$, the NE is also forced to deform by the movement of the cytoplasm. Thus, the CM and NE physically interact via the cytoplasm. Assuming that the cytoplasm is homogenous and isotropic, we simply express this interaction by a potential energy function $\Psi_{i j}$ with respect to the distance between the CM and NE. Mathematically, it is described as 
(a)

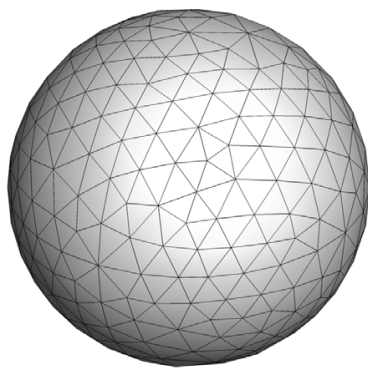

(b)

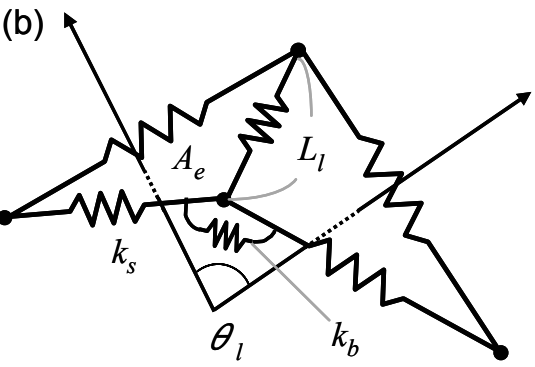

Fig. 2 (a) Appearance of CM and NE and (b) mechanical model of CM and NE.

(a)

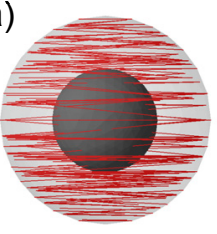

(b)

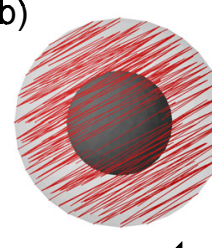

(c)

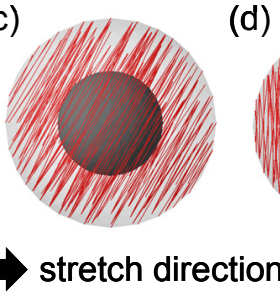

(d)

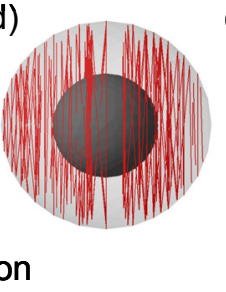

(e)

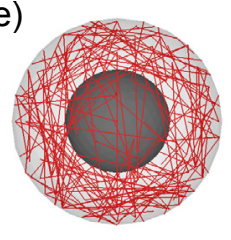

Fig. 3 Cell models with cytoskeletons of the initial orientation angle of (a) $0^{\circ}$, (b) $30^{\circ}$, (c) $60^{\circ}$, (d) $90^{\circ}$ and (e) at random. Red lines are cytoskeletons.

$$
\Psi_{i j}=k_{n}\left\{\frac{\pi y_{i j}}{2}-\tan \left(\frac{\pi y_{i j}}{2}\right)\right\} \quad\left(-1 \leq y_{i j} \leq 0\right)
$$

where $N_{n}{ }^{C M}$ and $N_{n}{ }^{N E}$ are the number of nodes on the CM and NE, respectively, $k_{n}$ is a parameter used to express the interaction between the $\mathrm{CM}$ and NE, $y_{i j}=\left(d_{i j}-\delta\right) / \delta, d_{i j}$ is the distance between node $i$ on the $\mathrm{CM}$ and $j$ on the NE, and $\delta$ is the radius difference between the $\mathrm{CM}$ and NE at their natural state. Note that $\Psi_{i j}$ was set to zero when $y_{i j}$ is larger than zero. This function generates a repulsive force for nodes on the $\mathrm{CM}$ and NE when their distance is smaller than the radius difference between the $\mathrm{CM}$ and $\mathrm{NE}$ at their natural state. This represents the situation where the NE and the CM are pushed each other through the movement of the cytoplasm when the CM comes closer to the NE. To the contrary, this function does not generate any forces even if node $j$ on the NE moves away from node $i$ on the CM. This is based on the consideration that the main component of cytoplasm is a cytosol that has highly-fluidic nature. Because of this nature, even if the NE moves away, it is considered that the cytoplasm flows in a room created by the movement of NE and the $\mathrm{CM}$ would not receive any attraction forces. For parameter settings and the influence of the parameter value on cell deformations, refer to Ujihara et $\mathrm{al}^{(16)}$.

An energy function for changes in the volume of the $\mathrm{CM}$ and $\mathrm{NE} W_{V}$ was also introduced as

$$
W_{V}=\frac{1}{2} k_{V} \frac{\left(V-V_{0}\right)^{2}}{V_{0}}
$$

where $k_{V}$ is the volume elasticity, $V$ is volume, and subscript 0 denotes the natural state. Refer to Ujihara et al. ${ }^{(16)}$ for the physical implications of eq. (10).

\subsection{Solving method}

Cell behavior is calculated by solving the motion equation of each node such that the total energy converges to the minimum at every time instant. For node $l$, the motion equation is given by

$$
m \ddot{\mathbf{r}}^{l}+c \dot{\mathbf{r}}^{l}=\mathbf{F}^{l}
$$


where a dot indicates a time derivative, $\mathbf{r}_{i}$ is the position vector of $i, m$ is mass, and $c$ is the artificial viscosity. Based on the virtual work theory, an elastic force $\mathbf{F}^{l}$ is calculated from

$$
\mathbf{F}^{l}=-\frac{\partial\left(W^{t}+W^{c}\right)}{\partial \mathbf{r}^{l}}
$$

where $W^{c}$ is the total energy stored in a cell;

$$
W^{c}=\sum_{\mathrm{CM}, \mathrm{NE}}\left(W_{s}+W_{b}+W_{A}+W_{V}\right)+W^{C S K}+\sum_{i=1}^{N_{n}^{C M}} \sum_{j=1}^{N_{n}^{N E}} \Psi_{i j}
$$

\subsection{Initial CSK orientation}

As shown in Fig. 3, five models that differed in initial CSK orientation, i.e., (a) $0^{\circ}$, (b) $30^{\circ}$, (c) $60^{\circ}$, (d) $90^{\circ}$ and (e) random orientations, with respect to the stretched direction of the tissue were prepared. For preparation of (a)-(d), 200 CSKs that satisfy certain criteria were chosen from all possible candidates of CSKs that were made by randomly connecting two nodes on the CM. The criteria were set as follows: (1) the CSK is longer than $4 \mu \mathrm{m}$ and (2) the orientation angle $\theta$ evaluated as an angle between each CSK and the stretched direction of the cell is within $6^{\circ}$. For preparation of (e), 200 combinations of two nodal points on the CM were randomly selected to create the model of randomly-oriented CSKs. Note that CSKs that penetrated a nucleus were not excluded in any cases.

\subsection{Simulation conditions}

The number of nodes and elements in a tissue was 3120 and 14610, respectively. The Poisson ratio $v$ was 0.45 , and the Young's modulus of a tissue $E^{t}$ was $10 \mathrm{kPa}$. A virtual mass on node $m$ of the tissue and CM was $1.0 \times 10^{-9} \mu \mathrm{g}$, and an artificial viscosity $c$ was $1.0 \times 10^{3}$ $\mu \mathrm{g} / \mathrm{s}$. In the cell models used, $N_{s}$ and $N_{b}$ were both 966 . CM and NE diameters were $20 \mu \mathrm{m}$ and $10 \mu \mathrm{m}$, respectively. For the CM, $k_{s}=7.0 \times 10^{6} \mu \mathrm{g} / \mathrm{s}^{2}, k_{b}=6.1 \times 10^{3} \mu \mathrm{g} \cdot \mu \mathrm{m} / \mathrm{s}^{2}, k_{A}=2.7 \times 10^{7}$ $\mu \mathrm{g} / \mathrm{s}^{2}, k_{a}=3.0 \times 10^{6} \mu \mathrm{g} / \mathrm{s}^{2}, k_{V}=5.0 \times 10^{6} \mu \mathrm{g} /\left(\mu \mathrm{m} \cdot \mathrm{s}^{2}\right)$, and $k_{n}=8.0 \times 10^{6} \mu \mathrm{g} \cdot \mu \mathrm{m} / \mathrm{s}^{2}$. The mass on the NE was set to half that on the CM, while other parameters for the NE were double of those for the CM. For CSK, $N^{C S K}=200, k_{C S K}=1.5 \times 10^{6} \mu \mathrm{g} / \mathrm{s}^{2}$. See Ujihara et al. ${ }^{(16)}$ for determining methods of these parameter values. The apparent Young's modulus of a cell $E^{c}$ was estimated by a linear regression analysis of the stress-strain data obtained from a numerical tensile test of a cell having the randomly-oriented cytoskeletons as described in Ujihara et $\mathrm{al}^{(16)}$. With the parameters described above, $E^{c}$ was estimated to be $1 \mathrm{kPa}$.

Stretch was applied to the tissue by moving nodes at $z=a$ in a direction of positive $z$ while fixing nodes at $z=-a$. During stretch, nodes at $z=-a$ and $a$ were constrained in the $z$ direction, but were free in $x$ and $y$ directions.

\subsection{Data analysis}

Deformations of a tissue, cell, and cytoskeletons were assessed with the following parameters. The tissue strain, $\varepsilon$ is calculated as

$$
\varepsilon^{t}=\frac{D}{D_{0}}-1
$$

where $D_{0}$ and $D$ are tissue length in the $z$ direction before and after deformation, respectively. Similarly, cell strain, $\varepsilon^{c}$ is defined as

$$
\varepsilon^{c}=\frac{d}{d_{0}}-1
$$

where $d_{0}$ and $d$ are cell diameter in the $z$ direction before and after deformation, respectively. Cytoskeletal strain $\varepsilon^{C S K}$ is simply gained by 


$$
\varepsilon^{C S K}=\frac{l}{l_{0}}-1
$$

where $l_{0}$ and $l$ are the length of a CSK before and after deformation, respectively. The number of cytoskeletons that are stretched and compressed is counted as $N_{s}^{C S K}$ and $N_{c}^{C S K}$. Moreover, the existence probability $P$ of cytoskeletal strain was calculated by counting the number of cytoskeletons having a specified strain at intervals of 0.003 between -0.15 and 0.15 , and dividing this by the total number of cytoskeletons, $N^{C S K}$.

\section{Results}

\subsection{Relationship between tissue strain and cell strain}

Figure 4 plots cell strain $\varepsilon^{c}$ against tissue strain $\varepsilon^{t}$ at the initial cytoskeleton orientation angles of $0^{\circ}, 30^{\circ}, 60^{\circ}$, and $90^{\circ}$ and that for the cell with randomly-oriented cytoskeletons under the condition $E^{t} / E^{c}=10$. A dashed line represents a unity of $\varepsilon^{c} / \varepsilon^{t}(=1)$. Regardless of the initial orientation angle of the cytoskeletons, the cell strain $\varepsilon^{c}$ increased monotonically as tissue strain $\varepsilon$ became larger. The same tendency was found for the cell with randomly-oriented cytoskeletons. The data of the randomly-oriented cytoskeletons lied behind the data of $\theta=60^{\circ}$.

Figure 5 plots the change in the strain ratio of cell to tissue against the initial

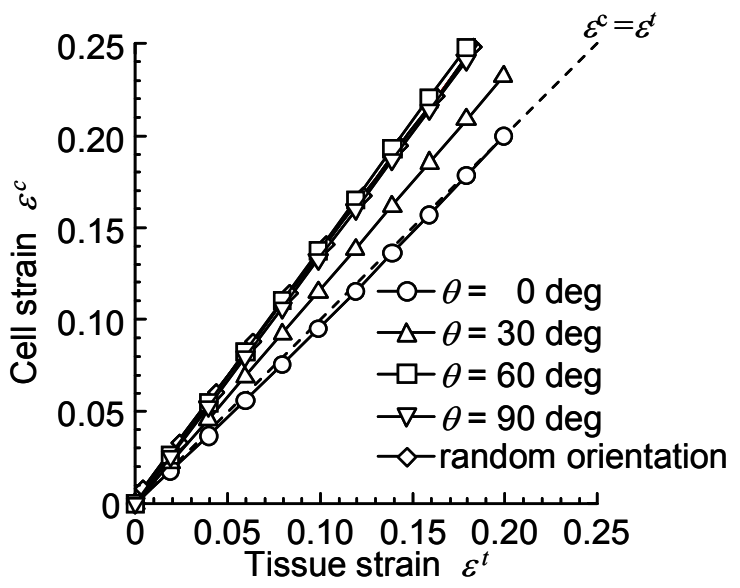

Fig. 4 Relationship between cell strain $\varepsilon^{c}$ and tissue strain $\varepsilon^{t}$ at the tissue subjected to uniaxial extension.

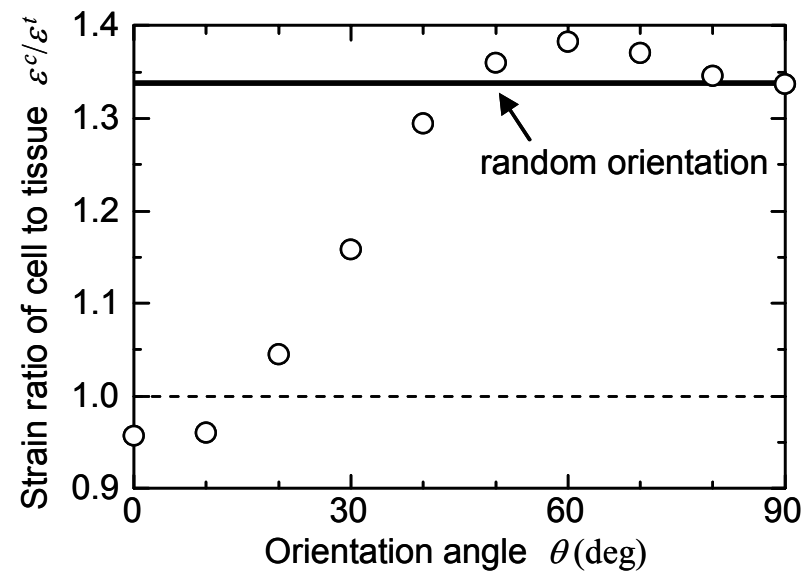

Fig. 5 Strain ratio of cell to tissue plotted against the cytoskeleton orientation angle. A dashed line represents a unity of the strain ratio. A black solid line represents the cell model with randomly-oriented cytoskeletons. 


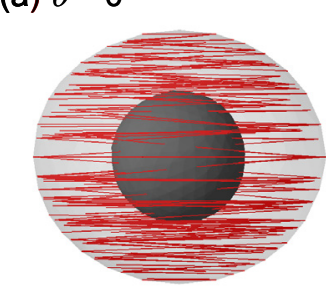

(b) $\theta=30^{\circ}$

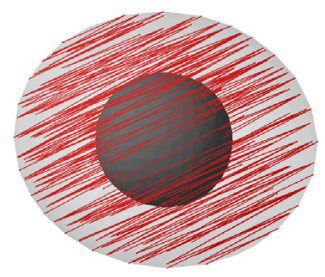

(c) $\theta=60^{\circ}$

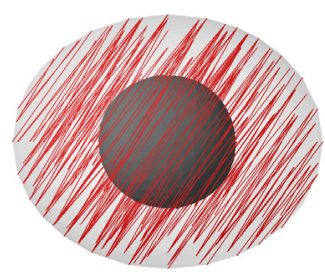

(d) $\theta=90^{\circ}$

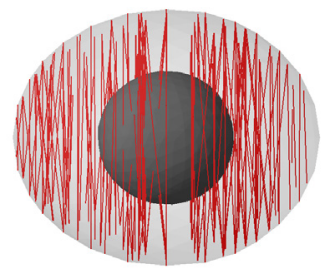

(e) random orientations

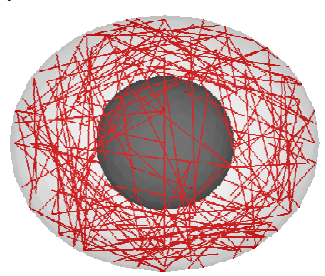

: cell has its own mechanical property
: cell has the same mechanical property as the tissue
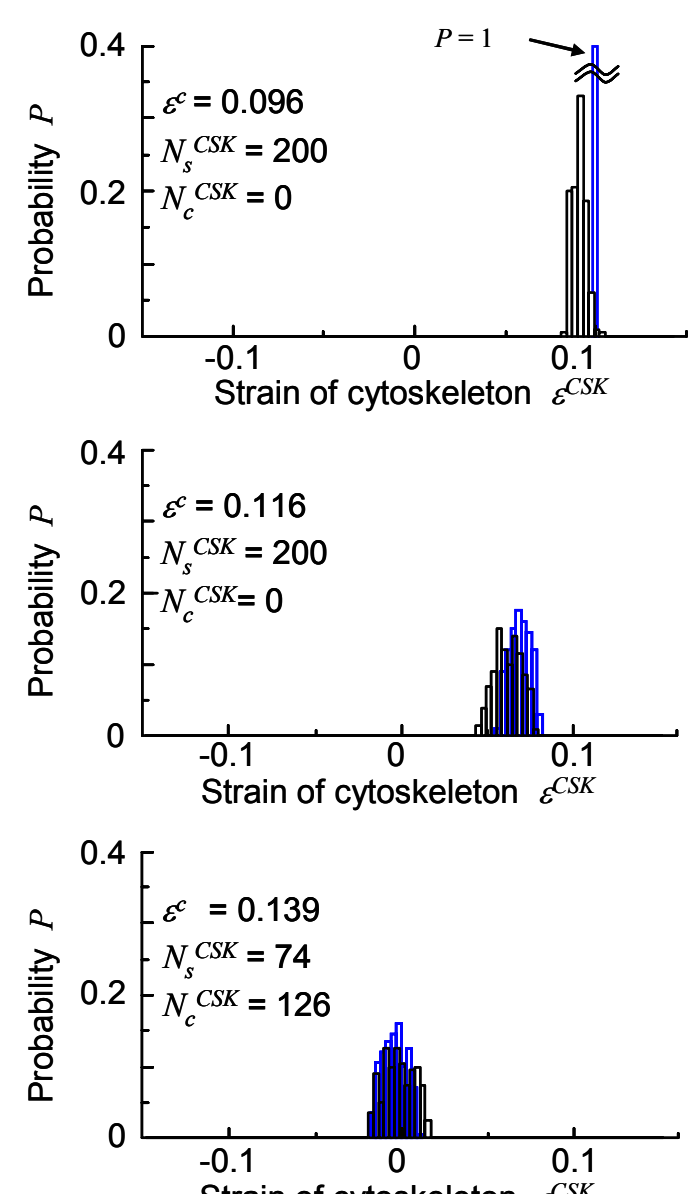

Strain of cytoskeleton $\varepsilon^{C S K}$
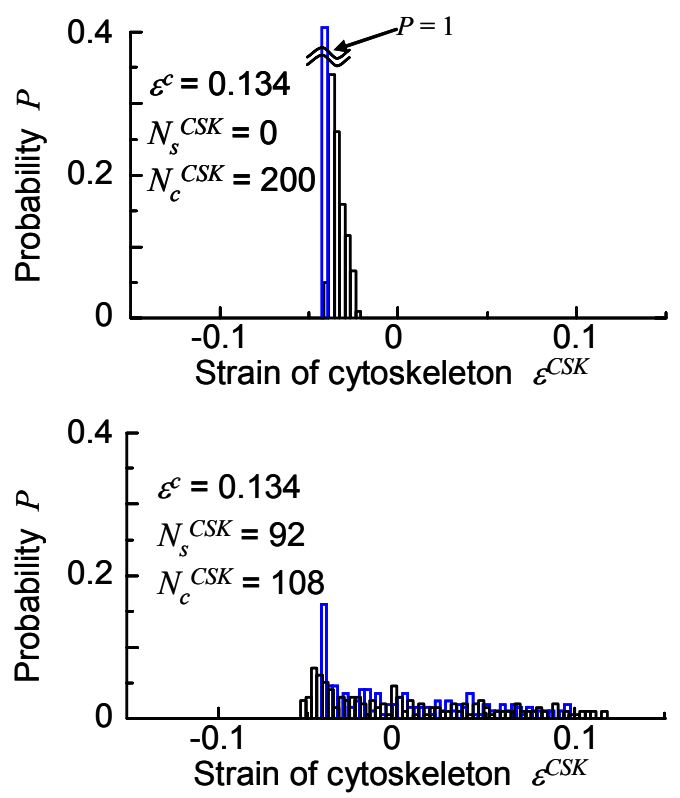

Fig. 6 Snapshots of a cell upon the tissue strain of 0.1. Probability distributions of cytoskeletal strain. (a) $\theta=$ $0^{\circ}$, (b) $30^{\circ}$, (c) $60^{\circ}$, (d) $90^{\circ}$, (e) randomly-oriented cytoskeletons. Black bars represent the data of the present computer simulations, whereas blue bars represent data obtained by considering a situation where the cell has the same mechanical property as the tissue. $N_{s}^{C S K}$ and $N_{c}^{C S K}$ are the number of stretched and compressed cytoskeletons obtained by the actual computer simulation where the cell has its own mechanical property. 


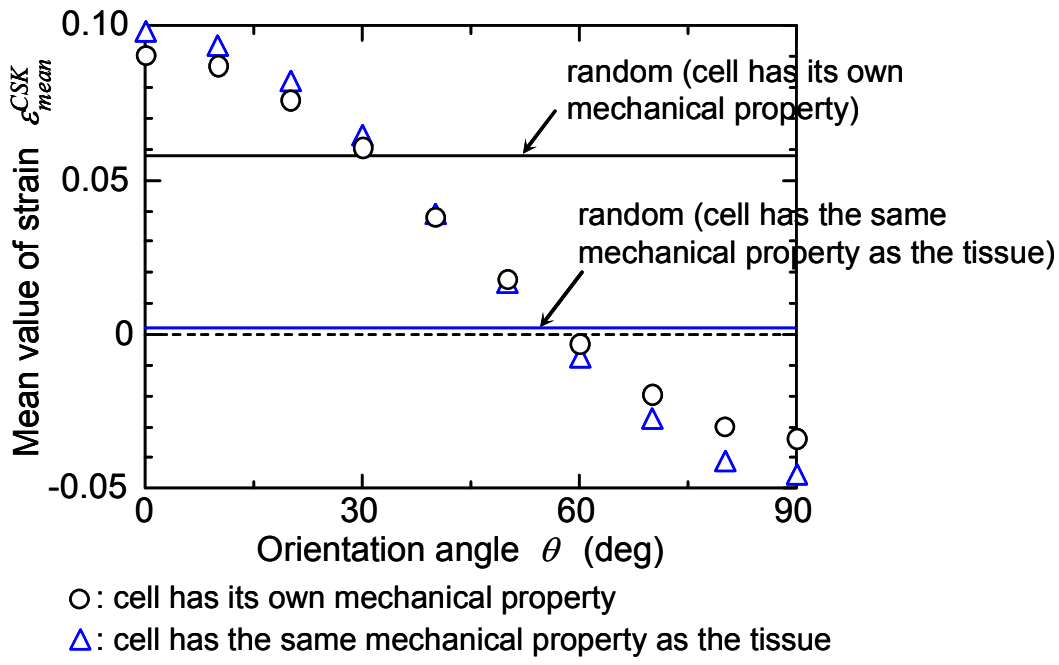

Fig. 7 Plot of mean cytoskeletal strain against cytoskeleton orientation angle. Black circles denote data of the present computer simulations where an interaction between the cell and tissue was considered, whereas blue triangles represent data obtained for the situation where the cell is assumed to have the same mechanical property as the tissue. The black and blue lines represent the mean value of strains of randomly-oriented cytoskeletons.

cytoskeleton orientation angle $\theta$ when the tissue is subjected to uniaxial extension of strain $\varepsilon^{t}=0.1$. A dashed line represents $\varepsilon^{c}=\varepsilon^{t}$. The strain ratio of cell to tissue was the smallest at $\theta=0^{\circ}$ and was lower than 1 when the initial orientation angle $\theta$ was smaller than $30^{\circ}$. Beyond $20^{\circ}$, the strain ratio rose monotonically with an increase in the initial orientation angle $\theta$, reaching a maximum at $\theta=60^{\circ}$ after crossing the line of the cell with randomly-oriented cytoskeletons. A subsequent increase in the initial orientation angle $\theta$ resulted in a decrease in a strain ratio of cell to tissue. The strain ratio at $\theta=90^{\circ}$ was almost the same as the one for the cell with randomly-oriented cytoskeletons.

\subsection{Effects of the initial cytoskeleton orientation angle on cytoskeletal strains}

Figure 6 presents snapshots of the cell and a probability distribution of cytoskeletal strains, obtained by the simulation, at initial cytoskeleton orientation angles of (a) $0^{\circ}$, (b) $30^{\circ}$, (c) $60^{\circ}$, (d) $90^{\circ}$ and (e) at random under the conditions of $E^{t} / E^{c}=10$ and $\varepsilon^{t}=0.1$ (see black bars). For comparison, each figure also presents the probability distribution of cytoskeletal strains obtained under the assumption that the cell has the same mechanical property as the tissue (the Poisson ratio of 0.45 , see blue bars). The method of calculating cytoskeletal strains for this ideal case is described in Appendix. The results showed a tendency that a larger orientation angle of cytoskeletons resulted in a larger deformation of a cell (see the value of $\varepsilon^{c}$ ), although the shape of a cell after deformation looked almost the same regardless of the initial orientation of cytoskeletons. A comparison of the histogram between black and blue bars demonstrated a small difference in their distribution. Overall, black ones were distributed more broadly than blue bars, indicating that cytoskeletons shown in black deformed more variously than the other because of a difference in the mechanical property between the cell and the tissue. In all cases except the case of the random orientation, the plot appeared to be a narrow Gaussian distribution. When the initial cytoskeleton orientation angle was $0^{\circ}$, the cytoskeletal strain ranged from 0.083 to 0.100 , suggesting that all cytoskeletons were stretched. The distribution shifted to the compression as the initial cytoskeleton orientation angle increased. Compressed cytoskeletons appeared at an initial cytoskeleton orientation angle of $60^{\circ}$. At this angle, the distribution was nearly 
symmetrical around a cytoskeletal strain of zero. In fact, the cytoskeletal strain ranged from -0.021 to 0.014 , with a mean of -0.003 . When the initial cytoskeleton orientation angle became $90^{\circ}$, all cytoskeletons were compressed. In the case of the random orientations, the histogram is broadly distributed. Interestingly, this case demonstrates a skewed distribution of cytoskeletal strains, in which stretched cytoskeletons showed a broad distribution, whereas compressed ones showed a narrow distribution. It is also noted that the maximum absolute value of tensile strain was larger than that of compressive strain in the case of randomly-oriented cytoskeletons.

Figure 7 depicts the relationship between the initial cytoskeleton orientation angle $\theta$ and the mean cytoskeletal strains obtained under the same condition as in Fig. 6. Black circles and a black solid line denote data obtained by the actual computer simulations where the cell has its own mechanical property, whereas blue triangles and a blue solid line represent data in which the cell is assumed to have the same mechanical property as the tissue. Here, the solid lines represent the mean value of strains of randomly-oriented cytoskeletons, and the broken line indicates a cytoskeletal strain of zero. As seen, the data obtained by the actual simulation showed qualitatively the same tendency as the results for the situation where the cell was assumed to have the same mechanical property as the tissue, although quantitatively, the computer simulations demonstrated an absolutely smaller value of the mean cytoskeletal strains than the other. The mean strain of randomly-oriented cytoskeletons obtained by the actual simulation was much larger than the other. In looking at the relationship between the mean cytoskeletal strain and the initial orientation angle of cytoskeletons, the mean cytoskeletal strain was maximal at an initial orientation angle of zero, and decreased monotonically with an increase in the initial orientation angle $\theta$. The trend curve crossed the line of zero strain at an initial orientation angle of around $60^{\circ}$, indicating there was almost no cytoskeletal deformation.

\section{Discussion}

Cytoskeletal strains were significantly dependent on cytoskeleton orientation angle (Fig. $6,7)$. When $\theta$ was $60^{\circ}$ and $90^{\circ}$, cytoskeletal strains were partially negative. This was attributed to Poisson's effect, by which a cell that was stretched in one-direction shrank in the transverse direction. Thus, even if a uniaxial stretch is applied to a tissue and a cell, some cytoskeletons undergo compression.

Cell strain was not the same as tissue strain, and varied significantly depending on the initial cytoskeleton orientation angle $\theta$ as demonstrated in Figs. 5 and 7. At $\theta=0$, the cell strain was minimal (Fig. 5), but cytoskeletal strain was maximal (Fig. 7). This was simply attributable to the fact that the cytoskeleton alignment was in the stretched direction of the cell, raising mechanical resistance against the stretch ${ }^{(11),(17)}$. In contrast, as mentioned earlier, at $\theta=60^{\circ}$, the cytoskeletal strain was nearly zero, indicating that cytoskeletons barely resisted cell deformation. Consequently, the cell strain was maximal at this angle. These data suggest that the strain of a cell residing in a tissue is significantly influenced by cytoskeleton orientation.

As shown in Fig. 6, the probability distribution of cytoskeletal strains obtained by the actual simulation was not the same as that obtained in an ideal situation where the cell has exactly the same mechanical property as the tissue. Moreover, Fig. 7 demonstrated a difference in the mean cytoskeletal strain between them. These differences are attributable to the difference in the mechanical property between the cell and the tissue, which induced an interaction between them. Through the interaction, deformations of cytoskeletons are more diverse. Such a difference is expected to increase as a difference in their mechanical properties becomes larger. Thus, it would be important to take account of cell mechanics for the analysis of cytoskeletal deformations when a cell resides in a cultured tissue.

It has been reported that human aortic endothelial cells cultured on a silicone membrane 
under a cyclic stretch appear to orient in the direction with minimal substrate strain ${ }^{(4)}$. Based on such results, together with experimental observations that cytoskeletons orient along the major axis of a cell, it was hypothesized that cells organize their cytoskeletons to minimize cytoskeletal strain ${ }^{(4)}$. In contrast, cells residing in cultured tissues favorably align along the stretched direction of the tissue. Although this phenomenon had been explained by contact guidance on collagen fibrils ${ }^{(18),(19)}$, Eastwood et al. ${ }^{(20)}$ cultured cells in a collagen gel and reported that even though the orientation of the collagen fibrils was still random, cells oriented in the stretched direction of the gel. Based on this result, they inferred that cells align themselves with the direction of maximum strain; in effect, they hide from the perceived strain. Assuming that the orientation of cytoskeletons corresponds to the major axis of an aligned cell, the results obtained at the cytoskeletal angle $\theta$ of $0^{\circ}$ represent the situation examined in Eastwood et al. ${ }^{(20)}$ where the cytoskeletons are aligned in the same direction as the tissue stretch. As seen in Fig. 7, the cytoskeletal strain was maximal when the cytoskeletal angle $\theta$ was $0^{\circ}$. At the same time, as demonstrated in Fig. 5, the cell strain was minimal at the cytoskeletal angle $\theta$ of $0^{\circ}$. These results indicate that aligning of the cultured cells in parallel to a stretch direction of tissue gives a large strain to cytoskeletons but makes the cell strain smaller. This finding actually supports the hypothesis of Eastwood et al. ${ }^{(20)}$ that cells in cultured tissues organize cytoskeletons in the direction of the tissue stretch in order for maximizing cytoskeletal strains to elevate mechanical resistance against external mechanical stimuli for self-protection.

In the present study, a tissue was treated as an isotropic linear material, such as a gel, which may cap applicability of the present model to actual tissue engineering problems. In fact, even in gel, structural and mechanical anisotropy increases with tissue development and remodeling ${ }^{(20)}$. Moreover, intracellular components and structure would change with time. Although improvements in tissue modeling remain as a future task, the present model would be a good tool to obtain preliminary data for understanding the mechanisms underlying cellular remodeling in a collagen gel construct.

\section{Conclusions}

In this study, we established a model that accounts for multi-scale mechanics of a tissue, cell, and cytoskeleton. The results demonstrated that even if the same strain was applied to a tissue, the degree of cell deformation appeared to differ depending on the cytoskeletal orientation. The present multi-scale model may help in achieving structural integration across physical scales of biomechanical organization from cytoskeletons to tissues and provide valuable information from biological reactions at cellular levels to mechanical events at the tissue level.

\section{Appendix}

Cytoskeletal strains when the cell has the same mechanical property as the tissue are estimated by the following procedure. Suppose a cytoskeleton connecting points $\mathrm{P}\left(x_{P}, y_{P}\right.$, $\left.z_{P}\right)$ and $\mathrm{Q}\left(x_{Q}, y_{Q}, z_{Q}\right)$ on the $\mathrm{CM}$ at the natural state. When the tissue is stretched in the $z$-direction by strain of $\varepsilon_{z}$, strains in the $x$ and $y$ directions are expressed by

$$
\varepsilon_{x}=\varepsilon_{y}=-v \varepsilon_{z}
$$

where $\varepsilon_{x}$ and $\varepsilon_{y}$ are the strains in the $x$ and $y$ directions, respectively. Thus, after the tissue deformation, points $\mathrm{P}$ and $\mathrm{Q}$ move to $\mathrm{P}^{\prime}\left(x_{P^{\prime}}, y_{P^{\prime}}, z_{P^{\prime}}\right)$ and $\mathrm{Q}^{\prime}\left(x_{Q^{\prime}}, y_{Q^{\prime}}, z_{Q^{\prime}}\right)$ whose coordinates are given by $\left(\left(1+\varepsilon_{x}\right) x_{P},\left(1+\varepsilon_{y}\right) y_{P},\left(1+\varepsilon_{z}\right) z_{P}\right)$ and $\left(\left(1+\varepsilon_{x}\right) x_{Q},\left(1+\varepsilon_{y}\right) y_{Q},\left(1+\varepsilon_{z}\right) z_{Q}\right)$. Then, the cytoskeletal strain $\varepsilon^{C S K}$ is calculated from

$$
\varepsilon^{C S K}=\frac{\left|\mathrm{P}^{\prime} \mathrm{Q}^{\prime}\right|}{|\mathrm{PQ}|}-1
$$


where $|\mathrm{PQ}|$ denotes the distance between $\mathrm{P}$ and Q. In order to obtain Figs. 6 and 7, the tissue with the Poisson ratio of 0.45 was stretched by $\varepsilon_{z}$ of 0.1 as done in the actual simulation. Eq. (A2) is applied to cytoskeletons set as the initial condition of each simulation to gain Figs. 6 and 7 .

\section{Acknowledgements}

The authors acknowledge receipt of a Grant-in-Aid for Scientific Research (B) 22300155 from the Ministry of Education, Culture, Sports, Science and Technology (MEXT).

\section{References}

(1) Wang, J.H. and Thampatty B.P., An introductory review of cell mechanobiology, Biomechanics and Modeling Mechanobiology, Vol. 5 (2006), pp. 1-16

(2) Sato, M., Ohshima, N. and Nerem, R.M., Viscoelastic properties of cultured porcine aortic endothelial cells, Journal of Biomechanics, Vol. 29 (1996), pp. 461-467.

(3) Kaunas, R., Nguyen, P., Usami, S. and Chien, S., Cooperative effects of Rho and mechanical stretch on stress fiber organization, Proceedings of National Academy Science of U. S. A., Vol. 102 (2005), pp. 15895-15900.

(4) Wang, J.H., Goldschmidt-Clermont, P., Wille, J. and Yin, F.C., Specificity of endothelial cell reorientation in response to cyclic mechanical stretching, Journal of Biomechanics, Vol. 34 (2001), pp. 1563-1572.

(5) Sato, M., Levesque, M.J. and Nerem, R.M., Micropipette aspiration of cultured bovine aortic endothelial cells exposed to shear stress, Arteriosclerosis, Vol. 7 (1987), pp. 276-286.

(6) Adachi, T., Sato, K. and Tomita, Y. Directional dependence of osteoblastic calcium response to mechanical stimuli, Biomechanics and Modeling Mechanobiology, Vol. 2 (2003), pp. 73-82.

(7) Sato, K., Adachi, T., Matsuo, M. and Tomita Y., Quantitative evaluation of threshold fiber strain that induces reorganization of cytoskeletal actin fiber structure in osteoblastic cells, Journal of Biomechanics, Vol. 38 (2005), pp.1895-1901.

(8) Dartsch, P.C., Hammerle, H. and Betz, E., Orientation of cultured arterial smooth muscle cells growing on cyclically stretched substrates, Acta Anatomica, Vol. 125 (1986), pp. 108-113.

(9) Neidlinger-Wilke, C., Grood, E. S., Wang, J. H. Brand, R. A. and Claes, L. Cell alignment is induced by cyclic changes in cell length: studies of cells grown in cyclically stretched substrates, Journal of Orthopedic Research, Vol. 19 (2001), pp. 286-293.

(10) Hayakawa, K., Tatsumi, H. and Sokabe M., Actin stress fibers transmit and focus force to activate mechanosensitive channels, Journal of Cell Science, Vol. 121 (2008), pp. 496-503.

(11) Nagayama, K. and Matsumoto, T., Mechanical anisotropy of rat aortic smooth muscle cells decreases with their contraction (possible effect of actin filaments orientation), JSME International Journal Series C, Vol. 47, (2004), pp. 985-991.

(12) Webb, K., Hitchcock, R. W., Smeal, R. M., Li, W., Gray, S. D. and Tresco, P. A., Cyclic strain increases fibroblast proliferation, matrix accumulation, and elastic modulus of fibroblast-seeded polyurethane constructs, Journal of Biomechanics, Vol. 39 (2006), pp. 1136-1144.

(13) Garvin, J., Qi, J., Maloney, M. and Banes, A.J., Novel system for engineering bioartificial tendons and application of mechanical load, Tissue Engineering, Vol. 9 (2003), pp. 967-979. 
(14) Arnoczky, S.P., Lavagnino, M., Whallon, J.H. and Hoonjan, A. In situ cell nucleus deformation in tendons under tensile load; a morphological analysis using confocal laser microscopy, Journal of Orthopedic Research, Vol. 20 (2002), pp. 29-35.

(15) Screen, H.R.C., Lee, D.A., Bader, D.L. and Shelton, J.C., Development of a technique to determine strains in tendons using the cell nuclei, Biorheology, Vol. 40 (2003), pp. 361-368.

(16) Ujihara, Y., Nakamura, M., Miyazaki, H. and Wada, S. Proposed spring network cell model based on a minimum energy concept, Annals of Biomedical Engineering, Vol. 38 (2010), pp. 1530-1538.

(17) Ujihara, Y., Nakamura, M., Miyazaki, H. and Wada, S., Effects of the initial orientation of actin fibers on global tensile properties of cells, Journal of Biomechanical Science and Engineering, Vol. 5 (2010), pp.515-525.

(18) Guido S. and Tranquillo, R.T., A methodology for the systematic and quantitative study of cell contact guidance in oriented collagen gels. Correlation of fibroblast orientation and gel birefringence, Journal of Cell Science, Vol. 105 (1993), pp. 317-331.

(19) Wang, J.H., Yang, G. and Li, Z., Controlling cell responses to cyclic mechanical stretching, Annals of Biomedical Engineering, Vol. 33 (2005), pp. 337-342.

(20) Eastwood, M., Mudera, V.C., McGrouther, D.A. and Brown, R.A., Effect of precise mechanical loading on fibroblast populated collagen lattices: morphological changes, Cell Motility and the Cytoskeleton, Vol. 40 (1998), pp.13-21. 